\title{
Positive Solutions to a Fractional-Order Two-Point Boundary Value Problem with $p$-Laplacian Operator
}

\author{
Xiangshan Kong ${ }^{1}$ and Haitao $\mathrm{Li}^{2}$ \\ ${ }^{1}$ Basic Science Department, Qingdao Binhai University, Qingdao 266555, China \\ ${ }^{2}$ School of Control Science and Engineering, Shandong University, Jinan 250061, China \\ Correspondence should be addressed to Haitao Li; haitaoli09@gmail.com
}

Received 13 August 2013; Accepted 23 September 2013

Academic Editors: S. Cingolani, C. Gutiérrez, G. Lu, and M. McKibben

Copyright (C) 2013 X. Kong and H. Li. This is an open access article distributed under the Creative Commons Attribution License, which permits unrestricted use, distribution, and reproduction in any medium, provided the original work is properly cited.

\begin{abstract}
This paper systematically investigates positive solutions to a kind of two-point boundary value problem (BVP) for nonlinear fractional differential equations with $p$-Laplacian operator and presents a number of new results. First, the considered BVP is converted to an operator equation by using the property of the Caputo derivative. Second, based on the operator equation and some fixed point theorems, several sufficient conditions are presented for the nonexistence, the uniqueness, and the multiplicity of positive solutions. Finally, several illustrative examples are given to support the obtained new results. The study of illustrative examples shows that the obtained results are effective.
\end{abstract}

\section{Introduction}

Fractional differential equation has recently attracted many scholars' interest due to its wide applications [1-3] in engineering, technology, biology, chemical process, and so on. The first issue for the theory of fractional differential equations is the existence of solutions to kinds of boundary value problems (BVPs), which has been studied recently by many scholars, and lots of excellent results have been obtained [4-17] by means of fixed point theorems, upper and lower solutions technique, and so forth.

As an important branch of BVPs, $p$-Laplacian equation was firstly introduced in [18] to model the following turbulent flow in a porous medium:

$$
\left(\varphi_{p}\left(u^{\prime}(t)\right)\right)^{\prime}=f\left(t, u(t), u^{\prime}(t)\right),
$$

where $\Phi_{p}(s)=|s|^{p-2} s, p>1, \Phi_{p}^{-1}=\Phi_{q}$, and $1 / p+$ $1 / q=1$. Then, it was investigated in both integer-order BVPs $[19,20]$ and fractional BVPs [21-25]. In [21], Chen and Liu considered the antiperiodic boundary value problem of fractional differential equation with $p$-Laplacian operator and obtained the existence of one solution by using Schaefer's fixed point theorem under certain nonlinear growth conditions. Han et al. [22] investigated a class of fractional boundary value problem with $p$-Laplacian operator and boundary parameter and presented several existence results for a positive solution in terms of the boundary parameter. It is noted that although there exist several results on the existence of one solution to fractional p-Laplacian BVPs, there are, to our best knowledge, relatively few results on the nonexistence, the uniqueness, and the multiplicity of positive solutions to fractional $p$-Laplacian BVPs.

In this paper, we study the following two-point boundary value problem of nonlinear fractional differential equations with $p$-Laplacian operator:

$$
\begin{gathered}
D_{0+}^{\beta} \Phi_{p}\left(D_{0+}^{\alpha} x(t)\right)=f(t, x(t)), \quad t \in[0,1], \\
x(0)=\gamma x(1), \quad D_{0+}^{\alpha} x(0)=\eta D_{0+}^{\alpha} x(1),
\end{gathered}
$$

where $\Phi_{p}(s)=|s|^{p-2} s, p>1, \Phi_{p}^{-1}=\Phi_{q}, 1 / p+1 / q=1,0<\alpha$, $\beta \leq 1,1<\alpha+\beta \leq 2,0<\gamma, \eta<1, f:[0,1] \times[0,+\infty) \rightarrow$ $[0,+\infty)$, and $D_{0+}^{\alpha}$ is the Caputo derivative. We first convert BVP (2) into an equivalent operator equation by using the property of the Caputo derivative and then present some sufficient conditions for the nonexistence, the uniqueness, and the multiplicity of positive solutions to this problem. Finally, we give several illustrative examples to support our new results. 
The main contributions of this paper are as follows. (i) We systematically study the nonexistence, the uniqueness, and the multiplicity of positive solutions to BVP (2) and propose some techniques to deal with fractional p-Laplacian BVPs, which enriches this academic area. (ii) We present a sufficient condition for the existence of three positive solutions to BVP (2) by introducing a free constant to construct a proper concave functional. The main feature of this free constant is that one can weaken the conditions by regulating the free constant (see Remark 12).

Throughout this paper, we consider BVP (2) in the real Banach space $E=C[0,1]$ with the norm $\|x\|=$ $\max _{t \in[0,1]}|x(t)|$. Let $P=\{x \in E: x(t) \geq 0$, for all $t \in[0,1]\}$. Then, $P$ is a normal solid cone of $E$ with $P^{\circ}=\{x \in E: x(t)>$ 0 , for all $t \in[0,1]\}$. A solution $x(t) \in E$ is said to be a positive solution to BVP (2), if $x \in P$ and $x(t) \not \equiv 0$. We will study the existence of positive solutions to BVP (2) in $P$.

The rest of this paper is structured as follows. Section 2 contains some preliminaries on the Caputo derivative. Section 3 investigates the nonexistence, the uniqueness, and the multiplicity of positive solutions to BVP (2) and presents the main results of this paper. In Section 4 , three illustrative examples are worked out to support our obtained results.

\section{Preliminaries}

In this section, we give some necessary preliminaries on the Caputo derivative, which will be used in the sequel. For details, please refer to [1-3] and the references therein.

Definition 1 (see [3]). The Riemann-Liouville fractional integral of order $\alpha>0$ of a function $y:(0,+\infty) \rightarrow \mathbb{R}$ is given by

$$
I_{0+}^{\alpha} y(t)=\frac{1}{\Gamma(\alpha)} \int_{0}^{t}(t-s)^{\alpha-1} y(s) d s,
$$

provided that the right side is pointwise defined on $(0,+\infty)$.

Definition 2 (see [3]). The Caputo fractional derivative of order $\alpha>0$ of a continuous function $y:(0,+\infty) \rightarrow \mathbb{R}$ is given by

$$
D_{0+}^{\alpha} y(t)=\frac{1}{\Gamma(n-\alpha)} \int_{0}^{t} \frac{y^{(n)}(s)}{(t-s)^{\alpha-n+1}} d s,
$$

where $n$ is the smallest integer greater than or equal to $\alpha$, provided that the right side is pointwise defined on $(0,+\infty)$.

One can easily obtain the following property from the definition of the Caputo derivative.

Proposition 3 (see [3]). Let $\alpha>0$. Assume that $y, D_{0+}^{\alpha} y \in$ $L(0,1)$. Then, the following equality holds:

$$
I_{0+}^{\alpha} D_{0+}^{\alpha} y(t)=y(t)+C_{0}+C_{1} t+\cdots+C_{n-1} t^{n-1},
$$

for some $C_{i} \in \mathbb{R}, i=0,1, \ldots, n-1$, where $n$ is the smallest integer greater than or equal to $\alpha$.

\section{Main Results}

In this section, we first convert BVP (2) into an equivalent operator equation and then present some new results on the nonexistence, the uniqueness, and the multiplicity of positive solutions to BVP (2).

Firstly, we convert BVP (2) into an equivalent operator equation.

Theorem 4. Given that $h \in C[0,1]$, the unique solution of

$$
\begin{aligned}
& D_{0+}^{\beta} \Phi_{p}\left(D_{0+}^{\alpha} x(t)\right)=h(t), \quad t \in[0,1], \\
& x(0)=\gamma x(1), \quad D_{0+}^{\alpha} x(0)=\eta D_{0+}^{\alpha} x(1)
\end{aligned}
$$

is

$$
\begin{aligned}
x(t)= & I_{0+}^{\alpha} \Phi_{q}\left(I_{0+}^{\beta} h(t)+A h(t)\right)+B h(t) \\
= & \frac{1}{\Gamma(\alpha)} \int_{0}^{t}(t-s)^{\alpha-1} \Phi_{q} \\
& \left(\frac{1}{\Gamma(\beta)} \int_{0}^{s}(s-\tau)^{\beta-1} h(\tau) d \tau+A h(s)\right) d s
\end{aligned}
$$$$
+B h(t)
$$

where

$$
\begin{aligned}
A h(t)= & \left.\frac{\Phi_{p}(\eta)}{1-\Phi_{p}(\eta)} I_{0+}^{\beta} h(t)\right|_{t=1} \\
= & \frac{\Phi_{p}(\eta)}{1-\Phi_{p}(\eta)} \frac{1}{\Gamma(\beta)} \\
& \times \int_{0}^{1}(1-s)^{\beta-1} h(s) d s, \quad \forall t \in[0,1], \\
B h(t)= & \left.\frac{\gamma}{1-\gamma} I_{0+}^{\alpha} \Phi_{q}\left(I_{0+}^{\beta} h(t)+A h(t)\right)\right|_{t=1} \\
= & \frac{\gamma}{1-\gamma} \frac{1}{\Gamma(\alpha)} \\
& \times \int_{0}^{1}(1-s)^{\alpha-1} \Phi_{q} \\
& \left(\frac{1}{\Gamma(\beta)} \int_{0}^{s}(s-\tau)^{\beta-1} h(\tau) d \tau+A h(s)\right) d s, \quad \forall t \in[0,1] .
\end{aligned}
$$

Proof. Assume that $x(t)$ satisfies (6). Then, from Proposition 3 , we have

$$
\Phi_{p}\left(D_{0+}^{\alpha} x(t)\right)=I_{0+}^{\beta} h(t)+c_{0}, \quad c_{0} \in \mathbb{R} .
$$

From the boundary value condition $D_{0+}^{\alpha} x(0)=\eta D_{0+}^{\alpha} x(1)$, one can see that

$$
c_{0}=\left.\frac{\Phi_{p}(\eta)}{1-\Phi_{p}(\eta)} I_{0+}^{\beta} h(t)\right|_{t=1}=A h(t) .
$$


Thus, we have

$$
x(t)=I_{0+}^{\alpha} \Phi_{q}\left(I_{0+}^{\beta} h(t)+A h(t)\right)+c_{1}, \quad c_{1} \in \mathbb{R},
$$

which together with the boundary value condition $x(0)=$ $\gamma x(1)$ yields that

$$
c_{1}=\left.\frac{\gamma}{1-\gamma} I_{0+}^{\alpha} \Phi_{q}\left(I_{0+}^{\beta} h(t)+A h(t)\right)\right|_{t=1}=B h(t) .
$$

The proof is completed.

Lemma 5. Consider that $x(t) \in E$ is a solution to BVP (2), if and only if $x(t)=T x(t)$, where

$$
\begin{aligned}
& T x(t)=I_{0+}^{\alpha} \Phi_{q}\left(I_{0+}^{\beta} N x(t)+A N x(t)\right)+B N x(t) \\
& =\frac{1}{\Gamma(\alpha)} \\
& \times \int_{0}^{t}(t-s)^{\alpha-1} \Phi_{q} \\
& \left(\frac{1}{\Gamma(\beta)} \int_{0}^{s}(s-\tau)^{\beta-1} f(\tau, x(\tau)) d \tau\right. \\
& +\frac{\Phi_{p}(\eta)}{1-\Phi_{p}(\eta)} \frac{1}{\Gamma(\beta)} \\
& \left.\times \int_{0}^{1}(1-\tau)^{\beta-1} f(\tau, x(\tau)) d \tau\right) d s \\
& +\frac{\gamma}{1-\gamma} \frac{1}{\Gamma(\alpha)} \\
& \times \int_{0}^{1}(1-s)^{\alpha-1} \Phi_{q} \\
& \left(\frac{1}{\Gamma(\beta)} \int_{0}^{s}(s-\tau)^{\beta-1} f(\tau, x(\tau)) d \tau\right. \\
& +\frac{\Phi_{p}(\eta)}{1-\Phi_{p}(\eta)} \frac{1}{\Gamma(\beta)} \\
& \left.\times \int_{0}^{1}(1-\tau)^{\beta-1} f(\tau, x(\tau)) d \tau\right) d s,
\end{aligned}
$$

and $N x(t)=f(t, x(t))$.

Proof. The necessity is obvious. Next, we prove the sufficiency.

In fact, if $x(t)=T x(t)$, a straightforward calculation shows that $x(0)=\gamma x(1)$.
Since

$$
\begin{aligned}
D_{0+}^{\alpha} x(t)= & \Phi_{q}\left(I_{0+}^{\beta} N x(t)+A N x(t)\right) \\
=\Phi_{q}( & \frac{1}{\Gamma(\beta)} \int_{0}^{t}(t-\tau)^{\beta-1} f(\tau, x(\tau)) d \tau \\
& +\frac{\Phi_{p}(\eta)}{1-\Phi_{p}(\eta)} \frac{1}{\Gamma(\beta)} \\
& \left.\times \int_{0}^{1}(1-\tau)^{\beta-1} f(\tau, x(\tau)) d \tau\right),
\end{aligned}
$$

we have $D_{0+}^{\alpha} x(0)=\eta D_{0+}^{\alpha} x(1)$, and

$$
\begin{aligned}
D_{0+}^{\beta} \Phi_{p}\left(D_{0+}^{\alpha} x(t)\right) & =D_{0+}^{\beta} \Phi_{p}\left(\Phi_{q}\left(I_{0+}^{\beta} N x(t)+A N x(t)\right)\right) \\
& =D_{0+}^{\beta}\left(I_{0+}^{\beta} f(t, x(t))+A N x(t)\right) \\
& =f(t, x(t)) .
\end{aligned}
$$

Therefore, $x(t)$ is a solution to BVP (2).

In the following, we consider the nonexistence of positive solutions to BVP (2) and present the following result.

Theorem 6. Consider BVP (2). Assume that

$$
f(t, x) \leq\left(m_{1} x\right)^{p-1}, \quad \forall(t, x) \in[0,1] \times[0,+\infty),
$$

where $m_{1}<(1-\gamma) \Gamma(\alpha+1)\left(1-\Phi_{p}(\eta)\right)^{q-1}(\Gamma(\beta+1))^{q-1}$ is a positive constant. Then, BVP (2) has no positive solution.

Proof. In fact, if BVP (2) has a positive solution $x(t) \in P$ with $x(t) \neq 0$. Then, one can see from (16) that

$$
\begin{aligned}
|x(t)|= & |T x(t)| \\
= & I_{0+}^{\alpha} \Phi_{q}\left(I_{0+}^{\beta} N x(t)+A N x(t)\right)+B N x(t) \\
= & \frac{1}{\Gamma(\alpha)} \\
& \times \int_{0}^{t}(t-s)^{\alpha-1} \Phi_{q} \\
& \quad\left(\frac{1}{\Gamma(\beta)} \int_{0}^{s}(s-\tau)^{\beta-1} f(\tau, x(\tau)) d \tau\right. \\
& \quad+\frac{\Phi_{p}(\eta)}{1-\Phi_{p}(\eta)} \frac{1}{\Gamma(\beta)} \\
& \times \frac{\gamma}{1-\gamma} \frac{1}{\Gamma(\alpha)}
\end{aligned}
$$


4

ISRN Mathematical Analysis

$$
\begin{aligned}
& \times \int_{0}^{1}(1-s)^{\alpha-1} \Phi_{q} \\
& \left(\frac{1}{\Gamma(\beta)} \int_{0}^{s}(s-\tau)^{\beta-1} f(\tau, x(\tau)) d \tau\right. \\
& +\frac{\Phi_{p}(\eta)}{1-\Phi_{p}(\eta)} \frac{1}{\Gamma(\beta)} \\
& \left.\times \int_{0}^{1}(1-\tau)^{\beta-1} f(\tau, x(\tau)) d \tau\right) d s \\
& \leq m_{1}\|x\| \frac{1}{\Gamma(\alpha)} \\
& \times \int_{0}^{t}(t-s)^{\alpha-1} \Phi_{q} \\
& \left(\frac{1}{\Gamma(\beta)} \int_{0}^{s}(s-\tau)^{\beta-1} d \tau\right. \\
& +\frac{\Phi_{p}(\eta)}{1-\Phi_{p}(\eta)} \frac{1}{\Gamma(\beta)} \\
& \left.\times \int_{0}^{1}(1-\tau)^{\beta-1} d \tau\right) d s \\
& +m_{1}\|x\| \frac{\gamma}{1-\gamma} \frac{1}{\Gamma(\alpha)} \\
& \times \int_{0}^{1}(1-s)^{\alpha-1} \Phi_{q} \\
& \left(\frac{1}{\Gamma(\beta)} \int_{0}^{s}(s-\tau)^{\beta-1} d \tau\right. \\
& +\frac{\Phi_{p}(\eta)}{1-\Phi_{p}(\eta)} \frac{1}{\Gamma(\beta)} \\
& \left.\times \int_{0}^{1}(1-\tau)^{\beta-1} d \tau\right) d s \\
& =m_{1}\|x\| \frac{1}{\Gamma(\alpha)} \\
& \times \int_{0}^{t}(t-s)^{\alpha-1} \Phi_{q} \\
& \left(\frac{1}{\Gamma(\beta+1)} s^{\beta}+\frac{\Phi_{p}(\eta)}{1-\Phi_{p}(\eta)} \frac{1}{\Gamma(\beta+1)}\right) d s \\
& +m_{1}\|x\| \frac{\gamma}{1-\gamma} \frac{1}{\Gamma(\alpha)} \\
& \times \int_{0}^{1}(1-s)^{\alpha-1} \Phi_{q} \\
& \left(\frac{1}{\Gamma(\beta+1)} s^{\beta}+\frac{\Phi_{p}(\eta)}{1-\Phi_{p}(\eta)} \frac{1}{\Gamma(\beta+1)}\right) d s \\
& \begin{aligned}
\leq & m_{1}\|x\| \frac{1}{\Gamma(\alpha)} \\
& \times \int_{0}^{t}(t-s)^{\alpha-1} \Phi_{q}
\end{aligned} \\
& \left(\frac{1}{\Gamma(\beta+1)}+\frac{\Phi_{p}(\eta)}{1-\Phi_{p}(\eta)} \frac{1}{\Gamma(\beta+1)}\right) d s \\
& +m_{1}\|x\| \frac{\gamma}{1-\gamma} \frac{1}{\Gamma(\alpha)} \\
& \times \int_{0}^{1}(1-s)^{\alpha-1} \Phi_{q} \\
& \left(\frac{1}{\Gamma(\beta+1)}+\frac{\Phi_{p}(\eta)}{1-\Phi_{p}(\eta)} \frac{1}{\Gamma(\beta+1)}\right) d s \\
& =m_{1}\|x\| \frac{1}{\Gamma(\alpha)} \\
& \times\left[\int_{0}^{t}(t-s)^{\alpha-1} d s\right] \Phi_{q} \\
& \left(\frac{1}{\Gamma(\beta+1)}+\frac{\Phi_{p}(\eta)}{1-\Phi_{p}(\eta)} \frac{1}{\Gamma(\beta+1)}\right) \\
& +m_{1}\|x\| \frac{\gamma}{1-\gamma} \frac{1}{\Gamma(\alpha)} \\
& \times\left[\int_{0}^{1}(1-s)^{\alpha-1} d s\right] \Phi_{q} \\
& \left(\frac{1}{\Gamma(\beta+1)}+\frac{\Phi_{p}(\eta)}{1-\Phi_{p}(\eta)} \frac{1}{\Gamma(\beta+1)}\right) \\
& =m_{1}\|x\| \frac{1}{\Gamma(\alpha+1)} t^{\alpha} \Phi_{q} \\
& \left(\frac{1}{\Gamma(\beta+1)}+\frac{\Phi_{p}(\eta)}{1-\Phi_{p}(\eta)} \frac{1}{\Gamma(\beta+1)}\right) \\
& +m_{1}\|x\| \frac{\gamma}{1-\gamma} \frac{1}{\Gamma(\alpha)} \Phi_{q} \\
& \left(\frac{1}{\Gamma(\beta+1)}+\frac{\Phi_{p}(\eta)}{1-\Phi_{p}(\eta)} \frac{1}{\Gamma(\beta+1)}\right) \\
& \leq m_{1}\|x\| \\
& \times \frac{1}{\Gamma(\alpha+1)\left(1-\Phi_{p}(\eta)\right)^{q-1}(\Gamma(\beta+1))^{q-1}} \\
& +m_{1}\|x\| \\
& \times \frac{\gamma}{(1-\gamma) \Gamma(\alpha+1)\left(1-\Phi_{p}(\eta)\right)^{q-1}(\Gamma(\beta+1))^{q-1}}
\end{aligned}
$$




$$
\begin{aligned}
& \leq m_{1}\|x\| \\
& \quad \times \frac{1}{(1-\gamma) \Gamma(\alpha+1)\left(1-\Phi_{p}(\eta)\right)^{q-1}(\Gamma(\beta+1))^{q-1}} \\
& <\|x\|, \quad \forall t \in[0,1],
\end{aligned}
$$

which is a contradiction. Hence, BVP (2) has no positive solution.

Next, we study the existence of a unique positive solution to BVP (2). To this end, we need the following fixed point theorem [26].

Definition 7. Let $P$ be a solid cone in a real Banach space $E$, $T: P^{\circ} \rightarrow P^{\circ}$ an operator, and $0 \leq \theta<1$. Then, $T$ is called a $\theta$-concave operator if

$$
T(k u) \geq k^{\theta} T u, \quad \forall 0<k<1, u \in P^{\circ} .
$$

Lemma 8. Assume that $P$ is a normal solid cone in a real Banach space $E, 0 \leq \theta<1$, and $T: P^{\circ} \rightarrow P^{\circ}$ is a $\theta$-concave increasing operator. Then $T$ has only one fixed point in $P^{\circ}$.

Based on Lemma 8, we have the following result.

Theorem 9. Consider BVP (2). Assume that for all $t \in[0,1]$, $f:[0,1] \times(0,+\infty) \rightarrow(0,+\infty)$ is increasing in $x$, and there exists a constant $0 \leq \theta<1$ such that

$$
\begin{gathered}
f(t, k x) \geq k^{(p-1) \theta} f(t, x), \\
\forall(t, x) \in[0,1] \times(0,+\infty), \quad \forall 0<k<1 .
\end{gathered}
$$

Then, BVP (2) has a unique positive solution.

Proof. Since $f:[0,1] \times(0,+\infty) \rightarrow(0,+\infty)$, one can see that $T: P^{\circ} \rightarrow P^{\circ}$.

For any $x_{1}, x_{2} \in P^{\circ}$ with $x_{1} \leq x_{2}$, from the monotonicity of $f$ and $x^{q-1}$, we have

$$
T x_{2}(t)-T x_{1}(t) \geq 0, \quad \forall t \in[0,1] .
$$

Thus, $T$ is increasing in $P^{\circ}$.

Next, we prove that $T$ is a $\theta$-concave operator.

In fact, from (19), for any $0<k<1, x \in P^{\circ}$, it is easy to see that

$$
\begin{aligned}
& T(k x)(t) \\
& =\frac{1}{\Gamma(\alpha)}
\end{aligned}
$$

$$
\begin{aligned}
& \times \int_{0}^{t}(t-s)^{\alpha-1} \Phi_{q} \\
& \left(\frac{1}{\Gamma(\beta)} \int_{0}^{s}(s-\tau)^{\beta-1} f(\tau, k x(\tau)) d \tau\right. \\
& +\frac{\Phi_{p}(\eta)}{1-\Phi_{p}(\eta)} \frac{1}{\Gamma(\beta)} \\
& \left.\times \int_{0}^{1}(1-\tau)^{\beta-1} f(\tau, k x(\tau)) d \tau\right) d s \\
& +\frac{\gamma}{1-\gamma} \frac{1}{\Gamma(\alpha)} \\
& \times \int_{0}^{1}(1-s)^{\alpha-1} \Phi_{q} \\
& \left(\frac{1}{\Gamma(\beta)} \int_{0}^{s}(s-\tau)^{\beta-1} f(\tau, k x(\tau)) d \tau\right. \\
& +\frac{\Phi_{p}(\eta)}{1-\Phi_{p}(\eta)} \frac{1}{\Gamma(\beta)} \\
& \left.\times \int_{0}^{1}(1-\tau)^{\beta-1} f(\tau, k x(\tau)) d \tau\right) d s \\
& \geq k^{\theta} \frac{1}{\Gamma(\alpha)} \\
& \times \int_{0}^{t}(t-s)^{\alpha-1} \Phi_{q} \\
& \left(\frac{1}{\Gamma(\beta)} \int_{0}^{s}(s-\tau)^{\beta-1} f(\tau, x(\tau)) d \tau\right. \\
& +\frac{\Phi_{p}(\eta)}{1-\Phi_{p}(\eta)} \frac{1}{\Gamma(\beta)} \\
& \left.\times \int_{0}^{1}(1-\tau)^{\beta-1} f(\tau, x(\tau)) d \tau\right) d s \\
& +k^{\theta} \frac{\gamma}{1-\gamma} \frac{1}{\Gamma(\alpha)} \\
& \times \int_{0}^{1}(1-s)^{\alpha-1} \Phi_{q} \\
& \left(\frac{1}{\Gamma(\beta)} \int_{0}^{s}(s-\tau)^{\beta-1} f(\tau, x(\tau)) d \tau\right. \\
& +\frac{\Phi_{p}(\eta)}{1-\Phi_{p}(\eta)} \frac{1}{\Gamma(\beta)} \\
& \left.\times \int_{0}^{1}(1-\tau)^{\beta-1} f(\tau, x(\tau)) d \tau\right) d s \\
& =k^{\theta} T(x)(t),
\end{aligned}
$$

which implies that $T$ is a $\theta$-concave operator.

By Lemma 8, BVP (2) has a unique positive solution. 
Finally, we investigate the multiplicity of positive solutions to BVP (2).

We first recall the famous Leggett-Williams fixed point theorem [26].

Let $E=(E,\|\cdot\|)$ be a Banach space and $P \subset E$ be a cone on $E$. A continuous mapping $\omega: P \rightarrow[0,+\infty)$ is said to be a concave nonnegative continuous functional on $P$, if $\omega$ satisfies $\omega(\lambda x+(1-\lambda) y) \geq \lambda \omega(x)+(1-\lambda) \omega(y)$ for all $x, y \in P$ and $\lambda \in[0,1]$.

Let $a, b, d>0$ be constants. Define $P_{d}=\{x \in P:\|x\|<$ $d\}, \overline{P_{d}}=\{x \in P:\|x\| \leq d\}$, and $P(\omega, a, b)=\{x \in P: \omega(x) \geq$ $a,\|x\| \leq b\}$.

Lemma 10. Let $E=(E,\|\cdot\|)$ be a Banach space, $P \subset E$ a cone of $E$, and $c>0$ a constant. Suppose that there exists a concave nonnegative continuous functional $\omega$ on $P$ with $\omega(x) \leq\|x\|$ for all $x \in \overline{P_{c}}$. Let $T: \overline{P_{c}} \rightarrow \overline{P_{c}}$ be a completely continuous operator. Assume that there are numbers $a, b$, and $d$ with $0<$ $d<a<b \leq c$, such that

(i) $\{x \in P(\omega, a, b): \omega(x)>a\} \neq \emptyset$ and $\omega(T x)>a$ for all $x \in P(\omega, a, b)$;

(ii) $\|T x\|<d$ for all $x \in \overline{P_{d}}$;

(iii) $\omega(T x)>$ a for all $x \in P(\omega, a, c)$ with $\|T x\|>b$.

Then, $T$ has at least three fixed points $x_{1}, x_{2}$, and $x_{3}$ in $\overline{P_{c}}$. Furthermore, $x_{1} \in P_{a} ; x_{2} \in\{x \in P(\omega, a, c): \omega(x)>a\}$; $x_{3} \in \overline{P_{c}} \backslash\left(P(\omega, b, c) \cup \overline{P_{a}}\right)$.

Now, based on Lemma 10, we consider the existence of three positive solutions to BVP (2).

Let $\xi \in[0,1)$ be a constant. Define $\omega(x)=\min _{t \in[\xi, 1]} x(t)$, for all $x \in P$. Then, one can easily see that $\omega$ is a concave nonnegative continuous functional. We have the following result.

Theorem 11. Consider BVP (2). Suppose that $f \in C([0,1] \times$ $[0,+\infty) \rightarrow[0,+\infty))$. Assume that there exist positive constants $a$ and $c$ with $a<\eta(1-\xi)^{\beta(q-1)}\left[(1-\gamma) \xi^{\alpha}+\gamma(1-\xi)^{\alpha}\right] c$, such that

(H1) there exists a constant $0<m_{2} \leq(1-\gamma) \Gamma(\alpha+1)(\Gamma(\beta+$ $1))^{q-1}\left(1-\Phi_{p}(\eta)\right)^{q-1}$, such that

$f(t, x) \leq\left(m_{2} c\right)^{p-1}, \quad \forall(t, x) \in[0,1] \times[0, c] ;$

(H2) there exists a constant $(1-\gamma) \Gamma(\alpha+1)(\Gamma(\beta+1))^{q-1}$ $\left(1-\Phi_{p}(\eta)\right)^{q-1} / \eta(1-\xi)^{\beta(q-1)}\left[(1-\gamma) \xi^{\alpha}+\gamma(1-\xi)^{\alpha}\right] \leq$ $m_{3}<m_{2} c / a$, such that

$$
f(t, x)>\left(m_{3} a\right)^{p-1}, \quad \forall(t, x) \in[\xi, 1] \times[a, c] ;
$$

(H3) there exists a constant $0<m_{4}<(1-\gamma)^{p-1}(\Gamma(\alpha+1))^{p-1}$ $\Gamma(\beta+1)\left(1-\Phi_{p}(\eta)\right)$, such that

$$
\varlimsup_{x \rightarrow 0^{+}} \sup _{t \in[0,1]} \frac{f(t, x)}{x^{p-1}}=m_{4} .
$$

Then, BVP (2) has at least three positive solutions.
Proof. Let us divide the proof into 4 steps.

Step 1. By (H1) and Lemma 5, for any $x \in \overline{P_{c}}$, we have

$$
\begin{aligned}
|x(t)|= & |T x(t)| \\
= & I_{0+}^{\alpha} \Phi_{q}\left(I_{0+}^{\beta} N x(t)+A N x(t)\right)+B N x(t) \\
= & \frac{1}{\Gamma(\alpha)} \\
& \times \int_{0}^{t}(t-s)^{\alpha-1} \Phi_{q} \\
& \left(\frac{1}{\Gamma(\beta)} \int_{0}^{s}(s-\tau)^{\beta-1} f(\tau, x(\tau)) d \tau\right. \\
& +\frac{\Phi_{p}(\eta)}{1-\Phi_{p}(\eta)} \frac{1}{\Gamma(\beta)} \\
& \left.\quad \frac{\gamma}{1-\gamma} \frac{1}{\Gamma(\alpha)}(1-\tau)^{\beta-1} f(\tau, x(\tau)) d \tau\right) d s \\
& \int_{0}^{1} \frac{(1-s)^{\alpha-1} \Phi_{q}}{\gamma}
\end{aligned}
$$$$
\left(\frac{1}{\Gamma(\beta)} \int_{0}^{s}(s-\tau)^{\beta-1} f(\tau, x(\tau)) d \tau\right.
$$$$
+\frac{\Phi_{p}(\eta)}{1-\Phi_{p}(\eta)} \frac{1}{\Gamma(\beta)}
$$$$
\left.\times \int_{0}^{1}(1-\tau)^{\beta-1} f(\tau, x(\tau)) d \tau\right) d s
$$$$
\begin{aligned}
\leq & m_{2} c \frac{1}{\Gamma(\alpha)} \\
& \times \int_{0}^{t}(t-s)^{\alpha-1} \Phi_{q}
\end{aligned}
$$$$
\left(\frac{1}{\Gamma(\beta)} \int_{0}^{s}(s-\tau)^{\beta-1} d \tau\right.
$$$$
+\frac{\Phi_{p}(\eta)}{1-\Phi_{p}(\eta)} \frac{1}{\Gamma(\beta)}
$$$$
\left.\times \int_{0}^{1}(1-\tau)^{\beta-1} d \tau\right) d s
$$

$$
+m_{2} c \frac{\gamma}{1-\gamma} \frac{1}{\Gamma(\alpha)}
$$

$$
\begin{aligned}
\times \int_{0}^{1}(1-s)^{\alpha-1} \Phi_{q} \\
\quad\left(\frac{1}{\Gamma(\beta)} \int_{0}^{s}(s-\tau)^{\beta-1} d \tau\right. \\
\left.\quad+\frac{\Phi_{p}(\eta)}{1-\Phi_{p}(\eta)} \frac{1}{\Gamma(\beta)} \int_{0}^{1}(1-\tau)^{\beta-1} d \tau\right) d s
\end{aligned}
$$


ISRN Mathematical Analysis

7

$$
\begin{array}{rlrl}
= & m_{2} c \frac{1}{\Gamma(\alpha)} & \leq m_{2} c \\
& \times \int_{0}^{t}(t-s)^{\alpha-1} \Phi_{q} & \times \frac{1}{\Gamma(\alpha+1)\left(1-\Phi_{p}(\eta)\right)^{q-1}(\Gamma(\beta+1))^{q-1}} \\
& \left.+\frac{1}{\Gamma(\beta+1)} s^{\beta}+\frac{\Phi_{p}(\eta)}{1-\Phi_{p}(\eta)} \frac{1}{\Gamma(\beta+1)}\right) d s & \times \frac{\gamma}{(1-\gamma) \Gamma(\alpha+1)\left(1-\Phi_{p}(\eta)\right)^{q-1}(\Gamma(\beta+1))^{q-1}} \\
\left.+m_{2} c \frac{\gamma}{1-\gamma} \frac{1}{\Gamma(\alpha)}\right) & \leq m_{2} c \\
\quad \int_{0}^{1}(1-s)^{\alpha-1} \Phi_{q} & \times \frac{1}{(1-\gamma) \Gamma(\alpha+1)\left(1-\Phi_{p}(\eta)\right)^{q-1}(\Gamma(\beta+1))^{q-1}} \\
\left(\frac{1}{\Gamma(\beta+1)} s^{\beta}+\frac{\Phi_{p}(\eta)}{1-\Phi_{p}(\eta)} \frac{1}{\Gamma(\beta+1)}\right) d s & \leq c, \quad \forall t \in[0,1],
\end{array}
$$$$
\begin{aligned}
\leq & m_{2} c \frac{1}{\Gamma(\alpha)} \\
& \times \int_{0}^{t}(t-s)^{\alpha-1} \Phi_{q}
\end{aligned}
$$$$
\left(\frac{1}{\Gamma(\beta+1)}+\frac{\Phi_{p}(\eta)}{1-\Phi_{p}(\eta)} \frac{1}{\Gamma(\beta+1)}\right) d s
$$$$
+m_{2} c \frac{\gamma}{1-\gamma} \frac{1}{\Gamma(\alpha)}
$$$$
\times \int_{0}^{1}(1-s)^{\alpha-1} \Phi_{q}
$$$$
\left(\frac{1}{\Gamma(\beta+1)}+\frac{\Phi_{p}(\eta)}{1-\Phi_{p}(\eta)} \frac{1}{\Gamma(\beta+1)}\right) d s
$$$$
=m_{2} c \frac{1}{\Gamma(\alpha)}\left[\int_{0}^{t}(t-s)^{\alpha-1} d s\right] \Phi_{q}
$$$$
\left(\frac{1}{\Gamma(\beta+1)}+\frac{\Phi_{p}(\eta)}{1-\Phi_{p}(\eta)} \frac{1}{\Gamma(\beta+1)}\right)
$$$$
+m_{2} c \frac{\gamma}{1-\gamma} \frac{1}{\Gamma(\alpha)}\left[\int_{0}^{1}(1-s)^{\alpha-1} d s\right] \Phi_{q}
$$$$
\left(\frac{1}{\Gamma(\beta+1)}+\frac{\Phi_{p}(\eta)}{1-\Phi_{p}(\eta)} \frac{1}{\Gamma(\beta+1)}\right)
$$$$
=m_{2} c \frac{1}{\Gamma(\alpha+1)} t^{\alpha} \Phi_{q}
$$$$
\left(\frac{1}{\Gamma(\beta+1)}+\frac{\Phi_{p}(\eta)}{1-\Phi_{p}(\eta)} \frac{1}{\Gamma(\beta+1)}\right)
$$$$
+m_{2} c \frac{\gamma}{1-\gamma} \frac{1}{\Gamma(\alpha)} \Phi_{q}
$$$$
\left(\frac{1}{\Gamma(\beta+1)}+\frac{\Phi_{p}(\eta)}{1-\Phi_{p}(\eta)} \frac{1}{\Gamma(\beta+1)}\right)
$$

which implies that $\|T x\| \leq c$. Thus, $T: \overline{P_{c}} \rightarrow \overline{P_{c}}$.

Next, let us show that $T: \overline{P_{c}} \rightarrow \bar{P}_{c}$ is completely continuous.

By the continuity of $f$ and $u^{q-1}$, we can get that $T: \overline{P_{c}} \rightarrow$ $\overline{P_{c}}$ is continuous and $T\left(\overline{P_{c}}\right)$ is bounded. Moreover, there exists a constant $M>0$ such that $\left|I_{0+}^{\beta} N x(t)+A N x(t)\right| \leq M$, for all $x \in \overline{P_{c}}, t \in[0,1]$.

In addition, for any $t_{1}, t_{2} \in[0,1]$ and $x \in \overline{P_{c}}$, we have

$$
\begin{aligned}
& \left|T x\left(t_{2}\right)-T x\left(t_{1}\right)\right| \\
& =\left|I_{0+}^{\alpha} \Phi_{q}\left(I_{0+}^{\beta} N x(t)+A N x(t)\right)\right|_{t=t_{2}} \\
& \quad-\left.I_{0+}^{\alpha} \Phi_{q}\left(I_{0+}^{\beta} N x(t)+A N x(t)\right)\right|_{t=t_{2}} \mid \\
& =\frac{1}{\Gamma(\alpha)} \mid \int_{0}^{t_{2}}\left(t_{2}-s\right)^{\alpha-1} \Phi_{q}\left(I_{0+}^{\beta} N x(s)+A N x(s)\right) d s \\
& -\int_{0}^{t_{1}}\left(t_{1}-s\right)^{\alpha-1} \Phi_{q} \\
& =\frac{1}{\Gamma(\alpha)} \mid \int_{0}^{t_{1}}\left(\left(t_{2}-s\right)^{\alpha-1}-\left(t_{1}-s\right)^{\alpha-1}\right) \Phi_{q} \\
& +\int_{t_{1}}^{t_{2}}\left(t_{2}-s\right)^{\alpha-1} \Phi_{q} \\
& \left(I_{0+}^{\beta} N x(s)+A N x(s)\right) d s \\
& \left(I_{0+}^{\beta} N x(s)+A N x(s)\right) d s \mid
\end{aligned}
$$




$$
\begin{gathered}
\leq \frac{M^{q-1}}{\Gamma(\alpha)}\left\{\int_{0}^{t_{1}}\left[\left(t_{1}-s\right)^{\alpha-1}-\left(t_{2}-s\right)^{\alpha-1}\right] d s\right. \\
\left.\quad+\int_{t_{1}}^{t_{2}}\left(t_{2}-s\right)^{\alpha-1} d s\right\} \\
=\frac{M^{q-1}}{\Gamma(\alpha+1)}\left[t_{1}^{\alpha}-t_{2}^{\alpha}+2\left(t_{2}-t_{1}\right)^{\alpha}\right] \longrightarrow 0 \\
\left|t_{2}-t_{1}\right| \rightarrow 0 .
\end{gathered}
$$

Thus, the Arzela-Ascoli theorem guarantees that $T$ : $\overline{P_{c}} \rightarrow \overline{P_{c}}$ is compact. Therefore, $T: \overline{P_{c}} \rightarrow \overline{P_{c}}$ is completely continuous.

Step 2. Choose a constant $b=c$. Let $x_{0}(t)=(a+b) / 2$, for all $t \in[0,1]$. Then, $\omega\left(x_{0}\right)=(a+b) / 2>a$ and $\left\|x_{0}\right\|=(a+b) / 2<$ $b$. Thus, $x_{0} \in\{x \in P(\omega, a, b): \omega(x)>a\} \neq \emptyset$.

Now, let us prove that $\omega(T x)>a$ holds for all $x \in$ $P(\omega, a, b)$. In fact, $x \in P(\omega, a, b)$ implies that $a \leq x(t) \leq b$, for all $t \in[\xi, 1]$. For any $t \in[\xi, 1]$, one can obtain from (H2) that

$T x(t)$

$$
\begin{aligned}
& \geq I_{0+}^{\alpha} \Phi_{q}(A N x(t))+B N x(t) \\
& \geq \frac{1}{\Gamma(\alpha)} \\
& \times \int_{0}^{t}(t-s)^{\alpha-1} \Phi_{q} \\
& \left(\frac{\Phi_{p}(\eta)}{1-\Phi_{p}(\eta)} \frac{1}{\Gamma(\beta)}\right. \\
& \left.\times \int_{\xi}^{1}(1-\tau)^{\beta-1} f(\tau, x(\tau)) d \tau\right) d s \\
& +\frac{\gamma}{1-\gamma} \frac{1}{\Gamma(\alpha)} \\
& \times \int_{\xi}^{1}(1-s)^{\alpha-1} \Phi_{q} \\
& \left(\frac{\Phi_{p}(\eta)}{1-\Phi_{p}(\eta)} \frac{1}{\Gamma(\beta)}\right. \\
& \left.\times \int_{\xi}^{1}(1-\tau)^{\beta-1} f(\tau, x(\tau)) d \tau\right) d s \\
& >\frac{1}{\Gamma(\alpha)}
\end{aligned}
$$

$$
\begin{aligned}
\times \int_{0}^{t}(t-s)^{\alpha-1} \Phi_{q} \\
\quad\left(\frac{\Phi_{p}(\eta)}{1-\Phi_{p}(\eta)} \frac{1}{\Gamma(\beta)}\right. \\
\left.\quad \times \int_{\xi}^{1}(1-\tau)^{\beta-1}\left(m_{3} a\right)^{p-1} d \tau\right) d s
\end{aligned}
$$$$
+\frac{\gamma}{1-\gamma} \frac{1}{\Gamma(\alpha)}
$$$$
\times \int_{\xi}^{1}(1-s)^{\alpha-1} \Phi_{q}
$$$$
\left(\frac{\Phi_{p}(\eta)}{1-\Phi_{p}(\eta)} \frac{1}{\Gamma(\beta)}\right.
$$$$
\left.\times \int_{\xi}^{1}(1-\tau)^{\beta-1}\left(m_{3} a\right)^{p-1} d \tau\right) d s
$$$$
=\frac{m_{3} a}{\Gamma(\alpha+1)} t^{\alpha}\left(\frac{\Phi_{p}(\eta)}{1-\Phi_{p}(\eta)} \frac{1}{\Gamma(\beta+1)}(1-\xi)^{\beta}\right)^{q-1}
$$$$
+\frac{\gamma}{1-\gamma} \frac{m_{3} a}{\Gamma(\alpha+1)}(1-\xi)^{\alpha}
$$$$
\times\left(\frac{\Phi_{p}(\eta)}{1-\Phi_{p}(\eta)} \frac{1}{\Gamma(\beta+1)}(1-\xi)^{\beta}\right)^{q-1}
$$$$
\geq \frac{m_{3} a}{\Gamma(\alpha+1)} \xi^{\alpha}\left(\frac{\Phi_{p}(\eta)}{1-\Phi_{p}(\eta)} \frac{1}{\Gamma(\beta+1)}(1-\xi)^{\beta}\right)^{q-1}
$$$$
+\frac{\gamma}{1-\gamma} \frac{m_{3} a}{\Gamma(\alpha+1)}(1-\xi)^{\alpha}
$$$$
\times\left(\frac{\Phi_{p}(\eta)}{1-\Phi_{p}(\eta)} \frac{1}{\Gamma(\beta+1)}(1-\xi)^{\beta}\right)^{q-1}
$$$$
=\frac{\left(\Phi_{p}(\eta)\right)^{q-1}(1-\xi)^{\beta(q-1)}\left((1-\gamma) \xi^{\alpha}+\gamma(1-\xi)^{\alpha}\right)}{(1-\gamma) \Gamma(\alpha+1)(\Gamma(\beta+1))^{q-1}\left(1-\Phi_{p}(\eta)\right)^{q-1}}
$$$$
\times m_{3} a \geq a .
$$

Hence, condition (i) of Lemma 10 holds.

Step 3. It is easy to see from (H3) that for all $t \in[0,1]$, for all $0<\varepsilon<(1-\gamma)^{p-1}(\Gamma(\alpha+1))^{p-1} \Gamma(\beta+1)\left(1-\Phi_{p}(\eta)\right)-m_{4}$, there exists $\delta>0$, such that

$$
f(t, x)<\left(m_{4}+\varepsilon\right) x^{p-1}, \quad 0 \leq x<\delta
$$


Let $0<d<\min \{\delta, a\}$. Now, we prove that $\|T x\|<d$ for all $x \in \overline{P_{d}}=\{x \in P:\|x\| \leq d\}$. As a matter of fact, for all $x \in \overline{P_{d}}$, one can see that

$$
\begin{aligned}
& |x(t)|=|T x(t)| \\
& =I_{0+}^{\alpha} \Phi_{q}\left(I_{0+}^{\beta} N x(t)+A N x(t)\right)+B N x(t) \\
& =\frac{1}{\Gamma(\alpha)} \\
& \times \int_{0}^{t}(t-s)^{\alpha-1} \Phi_{q} \\
& \left(\frac{1}{\Gamma(\beta)} \int_{0}^{s}(s-\tau)^{\beta-1} f(\tau, x(\tau)) d \tau\right. \\
& +\frac{\Phi_{p}(\eta)}{1-\Phi_{p}(\eta)} \frac{1}{\Gamma(\beta)} \\
& \left.\times \int_{0}^{1}(1-\tau)^{\beta-1} f(\tau, x(\tau)) d \tau\right) d s \\
& +\frac{\gamma}{1-\gamma} \frac{1}{\Gamma(\alpha)} \\
& \times \int_{0}^{1}(1-s)^{\alpha-1} \Phi_{q} \\
& \left(\frac{1}{\Gamma(\beta)} \int_{0}^{s}(s-\tau)^{\beta-1} f(\tau, x(\tau)) d \tau\right. \\
& +\frac{\Phi_{p}(\eta)}{1-\Phi_{p}(\eta)} \frac{1}{\Gamma(\beta)} \\
& \left.\times \int_{0}^{1}(1-\tau)^{\beta-1} f(\tau, x(\tau)) d \tau\right) d s \\
& \leq\left(m_{4}+\varepsilon\right)^{q-1}\|x\| \frac{1}{\Gamma(\alpha)} \\
& \times \int_{0}^{t}(t-s)^{\alpha-1} \Phi_{q} \\
& \left(\frac{1}{\Gamma(\beta)} \int_{0}^{s}(s-\tau)^{\beta-1} d \tau\right. \\
& +\frac{\Phi_{p}(\eta)}{1-\Phi_{p}(\eta)} \frac{1}{\Gamma(\beta)} \\
& \left.\times \int_{0}^{1}(1-\tau)^{\beta-1} d \tau\right) d s \\
& +\left(m_{4}+\varepsilon\right)^{q-1}\|x\| \frac{\gamma}{1-\gamma} \frac{1}{\Gamma(\alpha)}
\end{aligned}
$$

$$
\begin{aligned}
\times \int_{0}^{1}(1-s)^{\alpha-1} \Phi_{q} \\
\left(\frac{1}{\Gamma(\beta)} \int_{0}^{s}(s-\tau)^{\beta-1} d \tau\right. \\
\quad+\frac{\Phi_{p}(\eta)}{1-\Phi_{p}(\eta)} \frac{1}{\Gamma(\beta)} \\
\left.\quad \times \int_{0}^{1}(1-\tau)^{\beta-1} d \tau\right) d s
\end{aligned}
$$$$
=\left(m_{4}+\varepsilon\right)^{q-1}\|x\| \frac{1}{\Gamma(\alpha)}
$$$$
\times \int_{0}^{t}(t-s)^{\alpha-1} \Phi_{q}
$$$$
\left(\frac{1}{\Gamma(\beta+1)} s^{\beta}+\frac{\Phi_{p}(\eta)}{1-\Phi_{p}(\eta)} \frac{1}{\Gamma(\beta+1)}\right) d s
$$$$
+\left(m_{4}+\varepsilon\right)^{q-1}\|x\| \frac{\gamma}{1-\gamma} \frac{1}{\Gamma(\alpha)}
$$$$
\times \int_{0}^{1}(1-s)^{\alpha-1} \Phi_{q}
$$$$
\left(\frac{1}{\Gamma(\beta+1)} s^{\beta}+\frac{\Phi_{p}(\eta)}{1-\Phi_{p}(\eta)} \frac{1}{\Gamma(\beta+1)}\right) d s
$$$$
\leq\left(m_{4}+\varepsilon\right)^{q-1}\|x\| \frac{1}{\Gamma(\alpha)}
$$$$
\times \int_{0}^{t}(t-s)^{\alpha-1} \Phi_{q}
$$$$
\left(\frac{1}{\Gamma(\beta+1)}+\frac{\Phi_{p}(\eta)}{1-\Phi_{p}(\eta)} \frac{1}{\Gamma(\beta+1)}\right) d s
$$$$
+\left(m_{4}+\varepsilon\right)^{q-1}\|x\| \frac{\gamma}{1-\gamma} \frac{1}{\Gamma(\alpha)}
$$$$
\times \int_{0}^{1}(1-s)^{\alpha-1} \Phi_{q}
$$$$
\left(\frac{1}{\Gamma(\beta+1)}+\frac{\Phi_{p}(\eta)}{1-\Phi_{p}(\eta)} \frac{1}{\Gamma(\beta+1)}\right) d s
$$$$
=\left(m_{4}+\varepsilon\right)^{q-1}\|x\| \frac{1}{\Gamma(\alpha+1)} t^{\alpha} \Phi_{q}
$$$$
\left(\frac{1}{\Gamma(\beta+1)}+\frac{\Phi_{p}(\eta)}{1-\Phi_{p}(\eta)} \frac{1}{\Gamma(\beta+1)}\right)
$$$$
+\left(m_{4}+\varepsilon\right)^{q-1}\|x\| \frac{\gamma}{1-\gamma} \frac{1}{\Gamma(\alpha)} \Phi_{q}
$$$$
\left(\frac{1}{\Gamma(\beta+1)}+\frac{\Phi_{p}(\eta)}{1-\Phi_{p}(\eta)} \frac{1}{\Gamma(\beta+1)}\right)
$$ 


$$
\begin{aligned}
\leq & \left(m_{4}+\varepsilon\right)^{q-1}\|x\| \\
& \times \frac{1}{\Gamma(\alpha+1)\left(1-\Phi_{p}(\eta)\right)^{q-1}(\Gamma(\beta+1))^{q-1}} \\
& +\left(m_{4}+\varepsilon\right)^{q-1}\|x\| \\
& \times \frac{\gamma}{(1-\gamma) \Gamma(\alpha+1)\left(1-\Phi_{p}(\eta)\right)^{q-1}(\Gamma(\beta+1))^{q-1}} \\
\leq & \left(m_{4}+\varepsilon\right)^{q-1} \\
& \times d \frac{1}{(1-\gamma) \Gamma(\alpha+1)\left(1-\Phi_{p}(\eta)\right)^{q-1}(\Gamma(\beta+1))^{q-1}} \\
< & d, \quad \forall t \in[0,1],
\end{aligned}
$$

which implies that $\|T x\|<d$. Thus, $\|T x\|<d$, for all $x \in \overline{P_{d}}$.

Step 4. Let us prove that $\omega(T x)>a$ holds for all $x \in P(\omega, a, c)$ with $\|T x\|>b$.

For $x \in P(\omega, a, c)$ with $\|T x\|>b$, we have $a \leq x(t) \leq c$, for all $t \in[\xi, 1]$. From (H2), one can see that

$$
\begin{aligned}
T x(t) \\
\geq I_{0+}^{\alpha} \Phi_{q}(A N x(t))+B N x(t) \\
\geq \frac{1}{\Gamma(\alpha)} \\
\quad \times \int_{\xi}^{t}(t-s)^{\alpha-1} \Phi_{q} \\
\quad+\frac{\gamma}{1-\gamma} \frac{1}{\Gamma(\alpha)}\left(\frac{\Phi_{p}(\eta)}{1-\Phi_{p}(\eta)} \frac{1}{\Gamma(\beta)} \int_{\xi}^{1}(1-\tau)^{\beta-1} f(\tau, x(\tau)) d \tau\right) d s \\
\quad \times \int_{\xi}^{1}(1-s)^{\alpha-1} \Phi_{q} \\
>\quad \frac{1}{\Gamma(\alpha)}\left(\frac{\Phi_{p}(\eta)}{1-\Phi_{p}(\eta)} \frac{1}{\Gamma(\beta)} \int_{\xi}^{1}(1-\tau)^{\beta-1} f(\tau, x(\tau)) d \tau\right) d s \\
\quad \times \int_{\xi}^{t}(t-s)^{\alpha-1} \Phi_{q} \\
\quad+\frac{\gamma}{1-\gamma} \frac{1}{\Gamma(\alpha)} \\
\quad\left(\frac{\Phi_{p}(\eta)}{1-\Phi_{p}(\eta)} \frac{1}{\Gamma(\beta)} \int_{\xi}^{1}(1-\tau)^{\beta-1}\left(m_{3} a\right)^{p-1} d \tau\right) d s
\end{aligned}
$$

$$
\begin{aligned}
& \times \int_{\xi}^{1}(1-s)^{\alpha-1} \Phi_{q} \\
& \quad\left(\frac{\Phi_{p}(\eta)}{1-\Phi_{p}(\eta)} \frac{1}{\Gamma(\beta)} \int_{\xi}^{1}(1-\tau)^{\beta-1}\left(m_{3} a\right)^{p-1} d \tau\right) d s \\
= & \frac{m_{3} a}{\Gamma(\alpha+1)} \xi^{\alpha}\left(\frac{\Phi_{p}(\eta)}{1-\Phi_{p}(\eta)} \frac{1}{\Gamma(\beta+1)}(1-\xi)^{\beta}\right)^{q-1} \\
& +\frac{\gamma}{1-\gamma} \frac{m_{3} a}{\Gamma(\alpha+1)}(1-\xi)^{\alpha} \\
& \times\left(\frac{\Phi_{p}(\eta)}{1-\Phi_{p}(\eta)} \frac{1}{\Gamma(\beta+1)}(1-\xi)^{\beta}\right)^{q-1} \\
= & \frac{\left(\Phi_{p}(\eta)\right)^{q-1}(1-\xi)^{\beta(q-1)}\left((1-\gamma) \xi^{\alpha}+\gamma(1-\xi)^{\alpha}\right)}{(1-\gamma) \Gamma(\alpha+1)(\Gamma(\beta+1))^{q-1}\left(1-\Phi_{p}(\eta)\right)^{q-1}}
\end{aligned}
$$$$
\times m_{3} a \geq a, \quad \forall t \in[\xi, 1] .
$$

Therefore, condition (iii) of Lemma 10 is satisfied.

To sum up, all conditions of Lemma 10 hold. By Lemma 10, BVP (2) has at least three positive solutions.

Remark 12. It is noted that (H2) is dependent of the selection on $\xi$. Specifically, to obtain a weaker condition, one can choose $\xi^{*} \in[0,1)$ which maximizes

$$
k(\xi)=\xi^{\alpha}(1-\xi)^{\beta(q-1)}(1-\gamma)+\gamma(1-\xi)^{\alpha+\beta(q-1)} .
$$

\section{Illustrative Examples}

In this section, we give three illustrative examples to support our new results.

Example 1. Consider the following BVP:

$$
\begin{gathered}
D_{0+}^{1 / 2} \Phi_{3 / 2}\left(D_{0+}^{2 / 3} x(t)\right)=\frac{\left(1+t^{2}\right) \sqrt{x(t)}}{20}, \quad t \in[0,1], \\
x(0)=\frac{1}{2} x(1), \quad D_{0+}^{2 / 3} x(0)=\frac{1}{2} D_{0+}^{2 / 3} x(1) .
\end{gathered}
$$

A simple calculation shows that $(1-\gamma) \Gamma(\alpha+1)(1-$ $\left.\Phi_{p}(\eta)\right)^{q-1}(\Gamma(\beta+1))^{q-1} \approx 0.0301$.

Set $m_{1}=1 / 100<0.0301$, then one can see that

$$
f(t, x)=\frac{\left(1+t^{2}\right) \sqrt{x}}{20} \leq \sqrt{\frac{x}{100}}, \quad \forall(t, x) \in[0,1] \times[0, \infty],
$$

which implies that (16) holds. By Theorem 6, BVP (32) has no positive solution.

Example 2. Consider the following BVP:

$$
\begin{gathered}
D_{0+}^{\beta} \Phi_{2}\left(D_{0+}^{\alpha} x(t)\right)=\left(1+t^{2}\right) \sqrt[4]{x(t)}, \quad t \in[0,1], \\
x(0)=\gamma x(1), \quad D_{0+}^{\alpha} x(0)=\eta D_{0+}^{\alpha} x(1),
\end{gathered}
$$




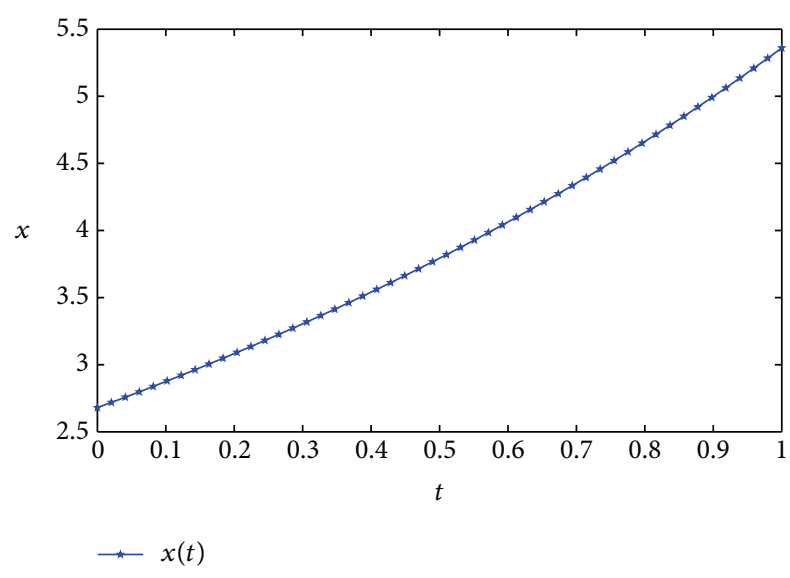

FIGURE 1: The unique positive solution to BVP (34) with $\alpha=\beta=1$ and $\gamma=\eta=1 / 2$.

where $0<\alpha, \beta \leq 1,1<\alpha+\beta \leq 2$, and $0<\gamma, \eta<1$ are arbitrary.

Set $\theta=1 / 4$; then it is easy to see that

$$
\begin{gathered}
f(t, k x)=\left(1+t^{2}\right) \sqrt[4]{k x}=k^{1 / 4} f(t, x), \\
\forall(t, x) \in[0,1] \times(0,+\infty), \quad \forall 0<k<1,
\end{gathered}
$$

Thus, (19) is satisfied. By Theorem 9, BVP (34) has a unique positive solution.

Let $\alpha=\beta=1$ and $\gamma=\eta=1 / 2$; we can draw the graph of the unique positive solution to BVP (34) by using MATLAB, shown in Figure 1.

Example 3. Consider the following BVP:

$$
\begin{gathered}
D_{0+}^{2 / 3} \Phi_{3}\left(D_{0+}^{1 / 2} x(t)\right)=f(t, x(t)), \quad t \in[0,1], \\
x(0)=\frac{1}{3} x(1), \quad D_{0+}^{1 / 2} x(0)=\frac{3}{4} D_{0+}^{1 / 2} x(1),
\end{gathered}
$$

where

$$
\begin{aligned}
& f(t, x) \\
& =\left(1+t^{2}\right) \begin{cases}\frac{x^{2}}{40}, & (t, x) \in[0,1] \times\left[0, \frac{1}{2}\right), \\
\frac{1599}{80} x-\frac{799}{80}, & (t, x) \in[0,1] \times\left[\frac{1}{2}, 1\right), \\
10, & (t, x) \in[0,1] \times[1,+\infty) .\end{cases}
\end{aligned}
$$

Set $a=1, c=50, \xi=0, m_{2}=1 / 5, m_{3}=2$, and $m_{4}=1 / 20$. By a straightforward calculation, one can easily see that (H1)(H3) hold. By Theorem 11, BVP (36) has at least three positive solutions.

\section{Acknowledgments}

The authors would like to thank the anonymous reviewers for their constructive comments and suggestions which improved the quality of the paper. This work is supported by the National Natural Science Foundation of China (G61174036), the Scholarship Award for Excellent Doctoral Student granted by Ministry of Education, and the Graduate Independent Innovation Foundation of Shandong University (yzc10064).

\section{References}

[1] K. B. Oldham and J. Spanier, The Fractional Calculus, Academic Press, London, UK, 1974.

[2] T. F. Nonnenmacher and R. Metzler, "On the Riemann-Liouvile fractional calculus and some recent applications," Fractals, vol. 3, pp. 557-566, 1995.

[3] S. G. Samko, A. A. Kilbas, and O. I. Marichev, Fractional Integrals and Derivatives, Theory and Applications, Gordon and Breach, Yverdon, Switzerland, 1993.

[4] X. Xu, D. Jiang, W. Hu, D. O’Regan, and R. P. Agarwal, "Positive properties of Green's function for threepoint boundary value problems of nonlinear fractional differential equations and its applications," Applicable Analysis, vol. 91, no. 2, pp. 323-343, 2012.

[5] A. Shi and S. Zhang, "Existence of positive solution to the Cauchy problem for a fractional diffusion equation with a singular nonlinearity," ISRN Mathematical Analysis, vol. 2012, Article ID 868295, 9 pages, 2012.

[6] Z. Bai, "On positive solutions of a nonlocal fractional boundary value problem," Nonlinear Analysis: Theory, Methods and Applications, vol. 72, no. 2, pp. 916-924, 2010.

[7] B. Ahmad and J. J. Nieto, "Existence results for a coupled system of nonlinear fractional differential equations with threepoint boundary conditions," Computers and Mathematics with Applications, vol. 58, no. 9, pp. 1838-1843, 2009.

[8] B. Ahmad and J. J. Nieto, "Boundary value problems for a class of sequential integrodifferential equations of fractional order," Journal of Function Spaces and Applications, vol. 2013, Article ID 149659, 8 pages, 2013.

[9] H. H. Alsulami, S. K. Ntouyas, and B. Ahmad, "Existence results for a Riemann-Liouville-type fractional multivalued problem with integral boundary conditions," Journal of Function Spaces and Applications, vol. 2013, Article ID 676045, 7 pages, 2013.

[10] D. Bǎleanu, O. G. Mustafa, and R. P. Agarwal, "An existence result for a superlinear fractional differential equation," Applied Mathematics Letters, vol. 23, no. 9, pp. 1129-1132, 2010.

[11] Z. Wei, W. Dong, and J. Che, "Periodic boundary value problems for fractional differential equations involving a RiemannLiouville fractional derivative," Nonlinear Analysis: Theory, Methods and Applications, vol. 73, no. 10, pp. 3232-3238, 2010.

[12] X. Yang, Z. Wei, and W. Dong, "Existence of positive solutions for the boundary value problem of nonlinear fractional differential equations," Communications in Nonlinear Science and Numerical Simulation, vol. 17, no. 1, pp. 85-92, 2012.

[13] Y. Zhao, S. Sun, Z. Han, and M. Zhang, "Positive solutions for boundary value problems of nonlinear fractional differential equations," Applied Mathematics and Computation, vol. 217, no. 16, pp. 6950-6958, 2011.

[14] Y. Zhao, S. Sun, Z. Han, and Q. Li, "The existence of multiple positive solutions for boundary value problems of nonlinear fractional differential equations," Communications in Nonlinear Science and Numerical Simulation, vol. 16, no. 4, pp. 2086-2097, 2011. 
[15] H. Li, X. Kong, and C. Yu, "Existence of three non-negative solutions for a three point boundary-value problem of nonlinear fractional differential equations," Electronic Journal of Differential Equations, vol. 2012, no. 88, pp. 1-12, 2012.

[16] A. Shi and S. Zhang, "Upper and lower solutions method and a fractional differential equation boundary value problem," Electronic Journal of Qualitative Theory of Differential Equations, vol. 2009, no. 30, pp. 1-13, 2009.

[17] S. Zhang, "Positive solutions for boundary-value problems of nonlinear fractional differential equations," Electronic Journal of Differential Equations, vol. 2006, no. 36, pp. 1-12, 2006.

[18] L. S. Leibenson, "General problem of the movement of a compressible fluid in a porous medium," Izvestiya Akademii Nauk Kirgizskoi SSR, vol. 9, pp. 7-10, 1983.

[19] H. Lv, D. O’Regan, and C. Zhang, "Multiple positive solutions for the one dimensional singular $p$-Laplacian," Applied Mathematics and Computation, vol. 133, pp. 407-422, 2002.

[20] Y. Wang and C. Hou, "Existence of multiple positive solutions for one-dimensional p-Laplacian," Journal of Mathematical Analysis and Applications, vol. 315, no. 1, pp. 144-153, 2006.

[21] T. Chen and W. Liu, "An anti-periodic boundaryvalue problem for the fractional differential equation with a $p$-Laplacian operator," Applied Mathematics Letters, vol. 25, pp. 1671-1675, 2012.

[22] Z. Han, H. Lu, S. Sun, and D. Yang, "Positive solutions to boundary-value problems of $p$-Laplacian fractional differential equations with a parameter in the boundary," Electronic Journal of Differential Equations, vol. 2012, no. 213, pp. 1-14, 2012.

[23] H. Lu and Z. Han, "Existence of positive solutions for boundaryvalue problem of fractional differential equation with $p$ laplacian operator," American Jouranl of Engineering and Technology Research, vol. 11, pp. 3757-3764, 2011.

[24] J. Wang and H. Xiang, "Upper and lower solutions method for a class of singular fractioal boundary-value problems with p-laplacian operator," Abstract and Applied Analysis, vol. 2010, Article ID 971824, 12 pages, 2010.

[25] X. Liu, M. Jia, and X. Xiang, "On the solvability of a fractional differential equation model involving the $p$-Laplacian operator," Computers and Mathematics with Applications, vol. 64, no. 10, pp. 3267-3275, 2012.

[26] D. Guo and V. Lakshmikantham, Nonlinear Problems in Abstract Cones, Academic Press, New York, NY, USA, 1988. 


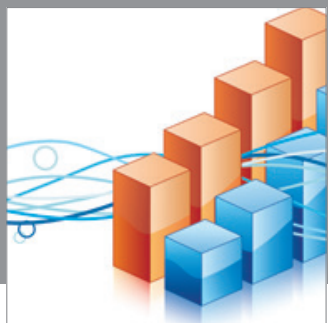

Advances in

Operations Research

mansans

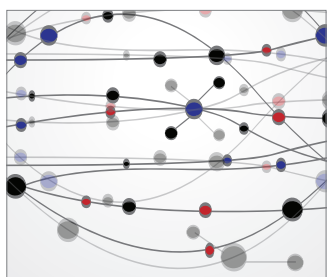

The Scientific World Journal
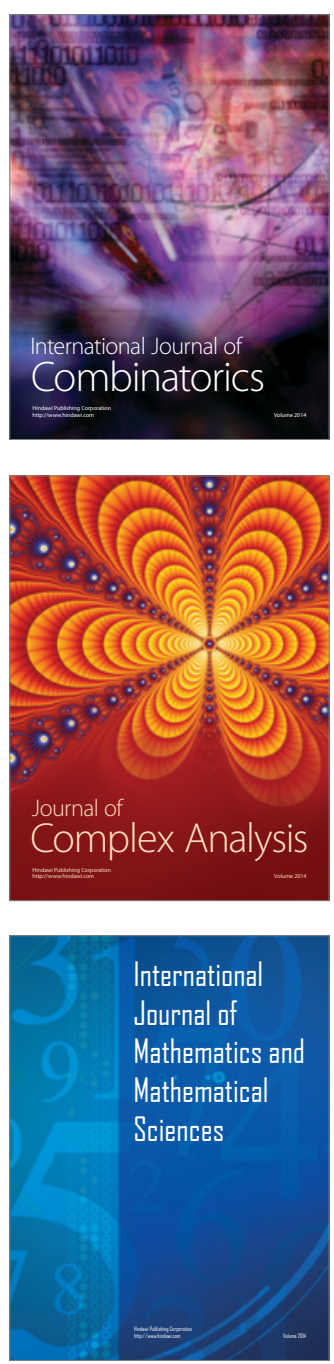
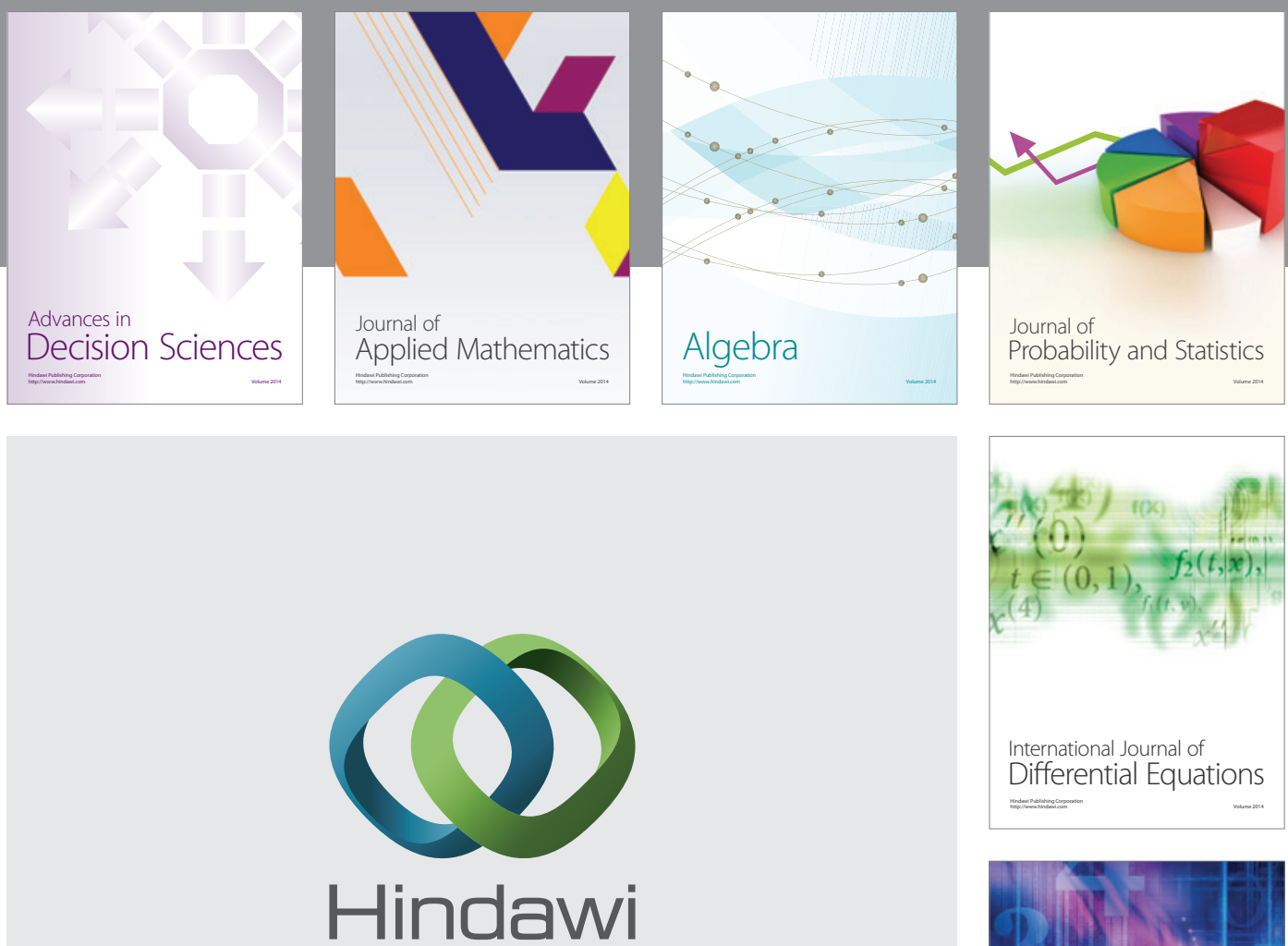

Submit your manuscripts at http://www.hindawi.com
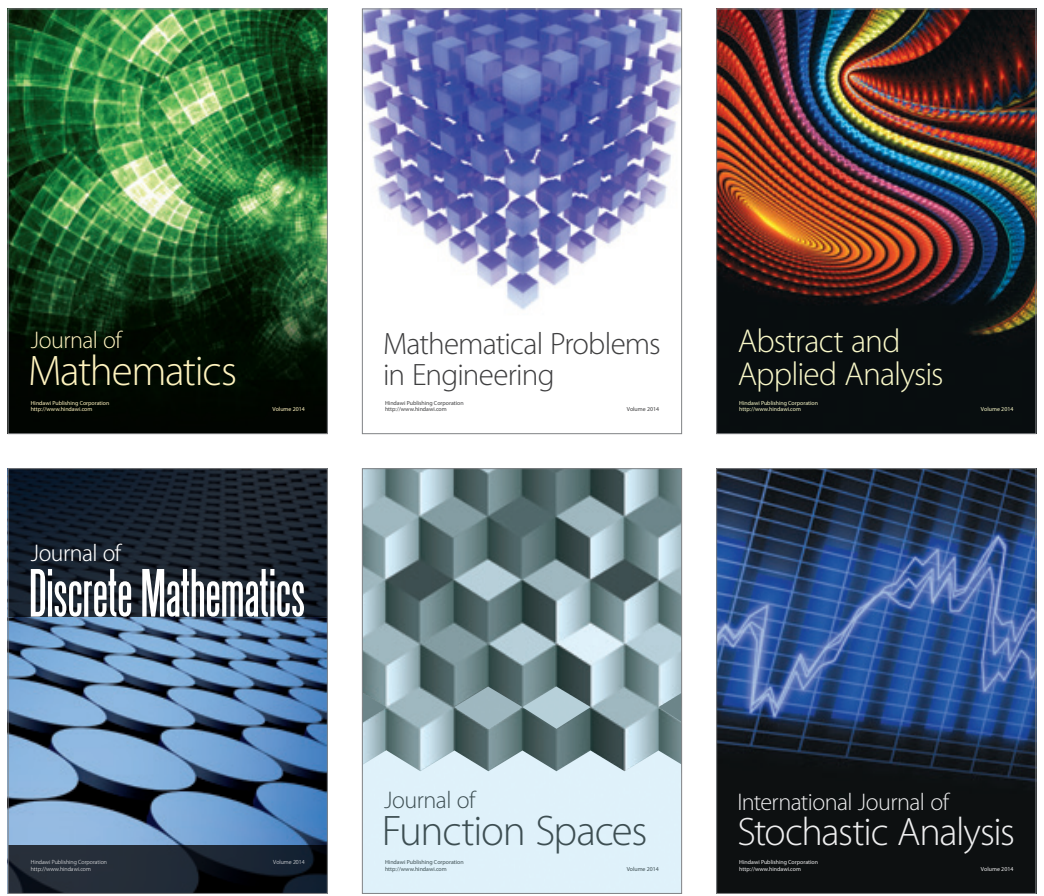

Journal of

Function Spaces

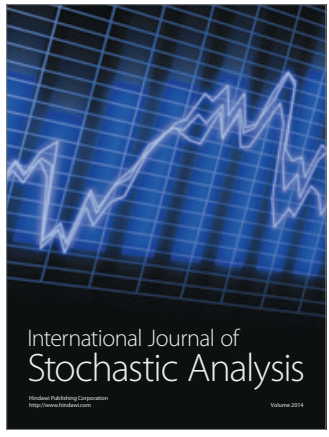

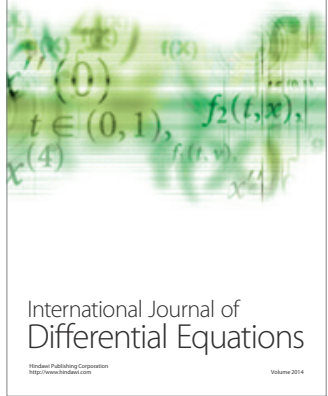
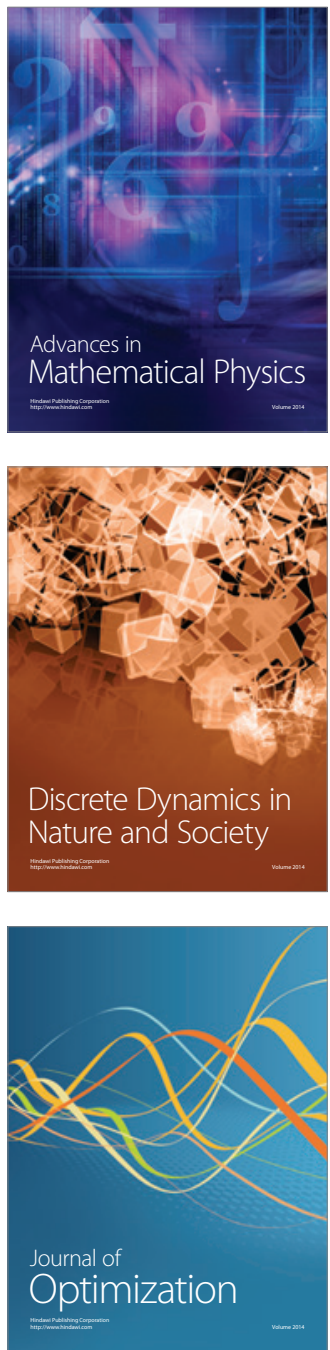